\title{
Stroke in patients with migraine
}

Jacques De Reuck ${ }^{1}$, Koen Paemeleire ${ }^{1}$, Georges Van Maele ${ }^{2}$

1Department of Neurology, Ghent University Hospital, Ghent, Belgium

2Biostatistical Unit, Ghent University Hospital, Ghent, Belgium

Neurologia i Neurochirurgia Polska 2010; 44, 2: 118-122

Correspondence address: Jacques De Reuck, Leopold II laan, 96, 9000 Ghent, Belgium, phone +32 9 221 88, fax +32 512440 10, e-mail: dereuck.j@gmail.com 


\section{Abstract}

Background and purpose: Migraine with aura (MA) is considered as a risk factor for ischaemic stroke. The present obser vational retrospective study compares migraine patients admitted for a documented stroke with those presenting focal neurological symptoms and headache without a demonstrable lesion and in which the final diagnosis was a migraine attack with aura.

Material and methods: The study included 14 migraine patients with a stroke and 37 without a stroke. The clinical characteristics, the vascular risk factors and the results of the technical examinations were compared.

Results: Stroke occurred in migraine patients with aura as well as without aura. Classical vascular risk factors were rather rare. Patent foramen ovale (PF0) with or without atrial septum aneurysm appeared to be the main risk factor for stroke in patients with a history of migraine. Infarcts were mainly located in the supratentorial territory of the posterior cerebral circulation. Also some lobar haematomas were observed, but their aetiology remained uncertain. The strokes were generally mildly severe with good outcome.

Hyperintense signals in the cerebral white matter and cerebellum, on $\mathrm{T}_{2}$-weighted magnetic resonance imaging, were more frequent in the migraine patients with stroke.

Conclusions: The presence of PFO, rather than of MA, appeared to be the main risk factor for stroke patients with migraine. No direct relation between migraine and stroke could be demonstrated.

Key words: migraine with aura, migraine without aura, posterior circulation infarct, lobar haematoma, patent foramen ovale, cerebral white matter changes. 
Migraine with aura (MA) is an established risk factor for ischaemic lesions of the brain, especially in women of childbearing age using contraceptives containing oestrogen [1]. There is also evidence that MA is linked to a broader range of ischaemic vascular disorders such as coronary and peripheral artery diseases [2]. In addition, individuals with migraine have a higher incidence of vascular risk factors, including arterial hypertension, diabetes and hyperlipidaemia [3]. However, migraine does not appear to predispose to atherosclerosis but is associated with a higher risk of venous thromboembolism [4]. Patent foramen ovale (PF0) appears to occur more frequently in patients with MA than in the general population and in migraine patients without aura (M0) [5].

Several hereditary conditions, such as cerebral autosomal dominant arteriopathy with subcortical infarcts and leukoencephalopathy (CADASIL), mitochondrial myopathy, encephalopathy, lactic acidosis and strokelike episodes (MELAS), and hereditary haemorrha gic telangiectasia appear to predispose to both migraine and stroke [6].

Clinical infarcts in patients with migraine are suggested to occur mainly in the posterior cerebral circulation, notably in the occipital lobes [7-13], although more recent studies stress a preferential location in the brainstem [14] and in the cerebellum [15].

However, both migraine and stroke are common and co-morbidity may, in some cases, be coincidental [16]. The present pilot study compares patients with a prior history of migraine with or without aura, who were admitted for an acute stroke or an episode of severe headache, accompanied by temporary focal neurological symptoms, in whom no cerebrovascular lesion was detected and in whom the final diagnosis was a migraine attack with aura.

Material and methods

This open observational retrospective study concerns 51 patients, out of a series of 1617 consecutively admitted to the Stroke Unit of the Ghent University Hospital between 2001 and 2007, who had a prior history of migraine and an episode of focal neurological deficit with or without severe headache. The patients were subdivided into 14 subjects with a documented stroke and 37 subjects in whom no cerebrovascular lesion was detected. 
The migraine patients were classified into those with a prior history of MA and those with M0, according to the "International Headache Classification" [17], apart from the episode leading to the final admission. The demographic features, including the vascular risk factors, were available in all patients. A family history investigation concerning migraine and stroke was only available in $51 \%$ of all patients. The degree of neurological impairment on admission, assessed with the National Institutes of Health Stroke Scale (NIHSS) [18], and the degree of disability on the modified Rankin scale (mRS) [19] at discharge, were compared between the two groups. All patients underwent neuroimaging of the brain: 69\% of the patients had computed tomography (CT) and $88 \%$ had magnetic resonance imaging (MRI) of the brain during admission, including $\mathrm{T}_{2}-$ and diffusion-weighted imaging. All patients had an extensive blood analysis, including rare blood clotting disorders. Mutations in the

NOTCH3 gene were only investigated in 3 patients with a history of migraine, stroke, severe white matter changes in the cerebral hemispheres on MRI and a fami ly history of migraine, stroke and dementia. A transoesophageal Doppler examination of the heart was performed in $71 \%$ of all patients, but in all of those who had a stroke. No transthoracic echography was done, as it is not sensitive enough to detect PFO.

No search for peripheral venous thrombosis was performed

in the patients with PF0. Conventional or MR angiography of the extra- and intracranial vessels was done in $96 \%$ of the total study population and in all stroke patients.

Statistical analysis was performed with $\mathrm{R}$ (a language and environment for statistical computing) [20]. Univariate comparison of unpaired groups was done with Fisher' s exact test for categorical data. The non-parametric Mann-Whitney $\boldsymbol{U}$-test was used to compare unpaired continuous variables. The significance level was set at $\alpha=0.05$, two-tailed.

Results

No statistical differences were observed between the stroke and the non-stroke patients concerning age $(p=0.45)$ and gender distribution $(p=0.20) \quad(78.6 \%$ and $56.8 \%$ were women, respectively). A history of MA was present in $42.9 \%$ of the stroke patients and in $51.4 \%$ of the non-stroke patients $(p=0.76)$. Headache accompanied stroke onset in $78.6 \%$ of patients.

A family history of migraine was present in $77.8 \%$ of 
the stroke group and in $50.0 \%$ of the non-stroke patients $(p=0.23)$. A family history of stroke was obtained in $22.2 \%$ and in $16.7 \%$, respectively $(p=1.00)$.

The incidence of classical age-related vascular risk factors was low in both groups and was not significantly different between them.

Transoesophageal Doppler examination showed presence of PF0 in $64.3 \%$ (35.7\% with an atrial septum aneurysm) of the stroke group while it was absent in the non-stroke patients $(p<0.001)$. The median NIHSS score was also low in both groups but was statistically different between groups $(p<0.001)$. The median mRS scores were also low and also significantly different between groups $(p<0.001)$ (Table 1).

Protein C deficiency was observed in $14.3 \%$ of the stroke patients, but was absent in the non-stroke group $(p=0.071)$. One stroke patient had significantly increased homocysteine plasma level of $37.2 \mu \mathrm{mol} / 1$. Mutations in the NOTCH3 gene were not observed in the 3 examined patients. Conventional or MR angiography showed only abnormalities in the former group $(p=0.020)$, consisting of carotid dissection in 1 patient, moderate atherosclerosis of the internal carotid artery in 1 patient and moderate atherosclerosis of a vertebral artery in another 1 patient.

In the stroke group, a lobar haematoma was found in $14.3 \%$ and ischaemic lesions in $85.7 \%$, of which 58. 3\% were located in the posterior and $41.7 \%$ in the anterior circulation.

A supratentorial infarct or haematoma was observed on CT and/or MRI in 13 out of the 14 stroke patients. In 1 patient a cerebellar infarct was observed. Additional small white matter T2 signals on MRI were seen in 42. 9\% of the stroke group and in $12.1 \%$ of the nonstroke group $(p=0.046)$. In the former group, $33.3 \%$ of the signals were present in the cerebellum, while they were not observed in the latter group $(p=0.50)$. Combining migraine history, female gender, use of oestrogen and smoking was found not to be statistically different between the groups: $14.3 \%$ of the stroke patients and $5.4 \%$ of the non-stroke ones $(p=0.30)$.

Discussion

This retrospective observational study investigates the occurrence of stroke in migraine patients who developed an episode of focal neurological disturbances. Due 
to the small number of patients the results have to be interpreted with some circumspection. We cannot

exclude that we have missed a migraine history in some patients admitted to our Stroke Unit, as the overall incidence was only $3.2 \%$. As the one-year prevalence of migraine in the general population is estimated to be about $6 \%$ in males and 15 to $18 \%$ in females [21], it is probable that some admitted patients forgot to mention prior migraine attacks, considered by them as not relevant. In the present study, a prior history of MA was not more frequent than of MO in the stroke patients, although this is considered as a significant risk factor $[22,23]$. We could also not demonstrate that young women with MA, using oestrogens and smoking, have an increased risk of stroke [24].

On the other hand, the predominance of infarcts in the supratentorial territory of the posterior circulation was confirmed [7-13,25]. The percentage incidence was $58.3 \%$, which is much higher than the $12.5 \%$ observed in the overall stroke population admitted to the Stroke Unit of the Ghent University Hospital [26]. Also two cases of temporal lobar haematoma were found. Both young patients had only arterial hypertension as a risk factor. No vascular malformation could be demonstrated on angiography.

Although a high incidence of PFO in patients with MA has been demonstrated [27], their causal relation remains uncertain [28]. Factors that may contribute to stroke in migraine include changes during cortical spreading depression with hyper- or hypoperfusion of neural tissue, vasospasm and endothelial dysfunction. Co-morbidity with PFO can be a mechanism of both disorders via a presumed lack of filtration of microemboli or toxic substances [29]. In the present study, $74.3 \%$ of the stroke patients had a PFO (half of them with an atrial septum aneurysm), while in the non-stroke group no PF0 was observed. Both groups consisted of young patients. Although the occurrence of PFO has been estimated to be between 20 and $25 \%$ in migraine patients without stroke [5], the difference with our stroke patients should still remain significant. The present study did not investigate the possibility of paradoxical emboli in the patients with stroke and PFO.

PFO and stroke were equally observed in patients with MA as with MO. Previous studies have shown that patients with migraine and a cryptogenic stroke have significantly larger PFOs than migraine patients without stroke [30]. Also patients with PFO associated 
with an atrial septum aneurysm have an increased risk of cerebral ischaemic events [31].

So, in our small series of migraine patients PF0 appears to be the major cause of cryptogenic ischaemic stroke. Carotid dissection has to be excluded as a rare cause. One older patient with an ischaemic stroke had hyperhomocysteinaemia as a possible vascular risk factor [32]. It remains uncertain what the cause of the lobar haematomas could be. The arterial hypertension of both young patients was well controlled and a cerebrovascular malformation could not be demonstrated on angiography. We could also show the presence of small T2-weighted hypersignals on MRI in the cerebral white matter and in the cerebellum, mainly in the migraine patients with a stroke $[15,33]$. Although the differences between the stroke and the non-stroke group were not statistically significant, T2-weighted hypersignals in the cerebellar white matter were only observed in the stroke patients with migraine, in addition to one patient with a documented cerebellar infarct. These white matter signals are not more frequent in migraine patients with PF0 [34]. They are not associated with cognitive deficits in middle-aged subjects and their significance remains unclear [35].

\section{Conclusion}

1. The present study demonstrates that PFO is the main risk factor for ischaemic stroke in migraine patients. 2. We found no evidence that MA itself was the cause of the strokes. 
Disclosure

Authors report no conflict of interest. 
1. Etminan M., Takkouche B., Isorna F.C., et al. Risk of ischaemic stroke in people with migraine: systematic review and metaanalysis of observational studies. BMJ 2005; 330: 63.

2. Kurth T., Schurks M., Logroscino G., et al. Migraine frequency and risk of cardiovascular disease in women. Neurology 2009; 73: 581-588.

3. Bigal M.E., Kurth T., Hu H., et al. Migraine and cardiovascular disease: possible mechanisms of interaction. Neurology 2009; 72: 1864-1871.

4. Schwaiger J., Kiechl S., Stockner H., et al. Burden of atherosclerosis and risk of venous thromboembolism in patients with

migraine. Neurology 2008; 71: 937-943.

5. Anzola G. P., Magoni M., Guindani M., et al. Potential source of cerebral embolism in migraine with aura: a transcranial Doppler study. Neurology 1999; 52: 1622-1625.

6. Tietjen G. E. The risk of stroke in patients with migraine and implications for migraine management. CNS Drugs 2005; 19: 683-692.

7. Broderick J.P., Swanson J.W. Migraine-related strokes. Clinical profile and prognosis in 20 patients. Arch Neurol 1987; 44: 868-871.

8. Bogousslavsky J., Regli F., Van Melle G., et al. Migraine stroke. Neurology 1988; 38: 223-227.

9. Rothrock J.F., Walicke P., Swenson M.R., et al. Migrainous stroke. Arch Neurol 1988; 45: 63-67.

10. Shuaib A., Lee M. A. Cerebral infarction in patients with migraine accompaniments. Headache 1988; 28: 599-601.

11. Sacquegna T., Andreoli A., Baldrati A., et al. Ischemic stroke in young adults: the relevance of migrainous infarction.

Cephalalgia 1989; 9: 255-258.

12. Caplan L. R. Migraine and vertebrobasilar ischemia. Neurology 1991; 41: 55-61.

13. Hoekstra-van Dalen R.A., Cillessen J.P., Kappelle L. J., et al. Cerebral infarcts associated with migraine: clinical features, risk factors and follow-up. J Neurol 1996; 243: 511-515.

14. Nardi K., Parnetti L., Pieri M. L., et al. Association between migraine and headache attributed to stroke: a case-control study. Headache 2008; 48: 1468-1475.

15. Kruit M. C., Launer L. J., Ferrari M. D., et al. Brain stem and cerebellar hyperintense lesions in migraine. Stroke 2006; 37:

1109-1112.

16. Tietjen G. E. Migraine as a systemic vasculopathy. Cephalgia 2009; 29: 987-996.

17. Headache Classification Committee of The International Headache Society. The International Classification of Headache Disorders. 2nd ed. Cephalalgia 2004; 24 (Supp1 1): 9-160.

18. Goldstein L. B., Bertels C., Davis J. N. Interrater reliability of the NIH stroke scale. Arch Neurol 1989; 46: 660-662.

19. Uyttenboogaart M., Luijckx G. J., Vroomen P. C., et al. Measuring disability in stroke: relationship between the modified Rankin scale and the Barthel index. J Neurol 2007; 254: 1113-1117.

20. R Development Core Team. R: A language and environment for statistical computing. R Foundation for Statistical Computing, Vienna 2007.

21. Bigal M.E., Lipton R.B. The epidemiology, burden, and comorbidities of migraine. Neurol Clin 2009; 27: 321-334.

22. Lampl C., Marecek S. Migraine and stroke - why do we talk 
about it? Eur J Neurol 2006; 13: 215-219.

23. Scher A. I., Gudmundsson L. S., Sigurdsson S., et al. Migraine headache in middle age and late-life brain infarcts. JAMA 2009; 301: 2563-2570.

24. Kurth T., Slomke M. A., Kase C. S., et al. Migraine, headache, and the risk of stroke in women: a prospective study. Neurology 2005; 64: 1020-1026.

25. Milhaud D., Bogousslavsky J., van Melle G., et al. Ischemic stroke and active migraine. Neurology 2001; 57: 1805-1811.

26. De Reuck J., Claeys I., Martens S., et al. Computed tomographic changes of the brain and clinical outcome of patients with seizures and epilepsy after an ischaemic hemispheric stroke. Eur

J Neurol 2006; 13: 402-407.

27. Marchione P., Ghiotto N., Sances G., et al. Clinical implications of patent foramen ovale in migraine with aura. Funct Neurol 2008;

23: 201-205.

28. Rundek T., Elkind M. S., Di Tullio M.R., et al. Patent foramen ovale and migraine: a cross-sectional study from the Northern Manhattan Study (NOMAS). Circulation 2008; 118: 1419-

1424 .

29. Elliott D. Migraine and stroke: current perspectives. Neurol Res 2008; 30: 801-812.

30. Anzola G.P., Morandi E., Casilli F., et al. Different degrees of right-to-left shunting predict migraine and stroke: data from

420 patients. Neurology 2006; 66: 765-767.

31. Mas J. L., Arquizan C., Lamy C., et al. Recurrent cerebrovascular events associated with patent foramen ovale, atrial septal aneurysm, or both. N Engl J Med 2001; 345: 1740-1746.

32. Moschiano F., D'Amico D., Usai S., et al. Homocysteine plasma levels in patients with migraine with aura. Neurol Sci2008; 29 (Suppl 1): S173-175.

33. Degirmenci B., Yaman M., Haktanir A., et al. Cerebral and cerebellar ADC values during a migraine attack. Neuroradiology 2007; 49: 419-426.

34. Adami A., Rossato G., Cerini R., et al. Right-to-left shunt does not increase white matter lesion load in migraine with aura patients. Neurology 2008; 71: 101-107.

35. Paemeleire K. Brain lesions and cerebral functional impairment in migraine patients. J Neurol Sci 2009; 283: 134-136. 
Table 1: Comparison of demographic and clinical features, and vascular risk factors between migraine patients with and without stroke

\begin{tabular}{|c|c|c|c|}
\hline Variable & $\begin{array}{l}\text { Patients with stroke } \\
\qquad(n=14)\end{array}$ & $\begin{array}{l}\text { Patients without stroke } \\
\qquad(n=37)\end{array}$ & P-value \\
\hline Median age (years); IQR & $46.0 ; 29.2-58.2$ & $40.0 ; 31.0-52.0$ & $0.447 *$ \\
\hline Women, \% & 78.6 & 56.8 & $0.20 *$ \\
\hline Migraine with aura, $\%$ & 42.9 & 51.4 & $0.76^{*}$ \\
\hline Family history of migraine, $\%$ & 77.8 & 50.0 & $0.23 *$ \\
\hline Family history of stroke, $\%$ & 22.2 & 16.7 & $1^{*}$ \\
\hline Median NIHSS score; IQR & $5.5 ; 2.2-7.8$ & $0.0 ; 0.0-0.0$ & $<0.001^{*}$ \\
\hline Median modified Rankin scale score; IQR & $2 ; 0-2$ & $0 ; 0-0$ & $<0.001^{*}$ \\
\hline Patent foramen ovale, $\%$ & 64.3 & 0 & $<0.001^{*}$ \\
\hline Arterial hypertension, \% & 35.7 & 10.8 & $0.09 *$ \\
\hline Coronary artery disease, $\%$ & 0 & 0 & - \\
\hline Atrial fibrillation, $\%$ & 0 & 0 & - \\
\hline Peripheral artery disease, $\%$ & 0 & 2.0 & $1 *$ \\
\hline Cardiac valvular disease, $\%$ & 0 & 2.7 & $1^{*}$ \\
\hline Hypercholesterolaemia, \% & 7.1 & 0 & $0.28 *$ \\
\hline Diabetes, $\%$ & 0 & 8.1 & $0.55^{*}$ \\
\hline Smoking, $\%$ & 14.3 & 16.2 & $1^{*}$ \\
\hline Oestrogen use, \% & 21.4 & 10.8 & $0.38^{*}$ \\
\hline
\end{tabular}

NIHSS - National Institutes of Health Stroke Scale; IQR - Inter Quartile Range

${ }^{*}$ Mann-Whitnev U-test 